\title{
MAGE-A3/HPV 16 Peptide Vaccine
}

National Cancer Institute

\section{Source}

National Cancer Institute. MAGE-A3/HPV 16 Peptide Vaccine. NCI Thesaurus. Code C61088.

A multi-epitope Trojan antigen (TA) construct vaccine consisting of human melanoma antigen A3 (MAGE-A3) and human papillomavirus (HPV) 16 peptide epitopes linked by the furin-sensitive linker peptide RVKR (arg inine-serine-lysine-arginine) with immunostimulatory and antitumor activities. The TA construct enters the cytoplasm of antigen-presenting cells (APC) and is processed by the endoplasmic reticulum (ER) and the trans-Golgi network (TGN), where the endopeptidase furin releases the epitopes from the RVKR linker peptide and, together with various exopeptidases, generates MHC class I-binding peptides. Expressed on the cell surfaces of APC, these MHC class I-binding peptides stimulate a cytotoxic T lymphocyte (CTL) response against tumor cells that display the same peptide epitopes on their cell surfaces. 